\title{
Remission of diffusion lesions in acute stroke magnetic resonance imaging
}

\author{
F. A. Fellner ${ }^{1}$, M. R. Vosko², C. M. Fellner ${ }^{1}$, D. Flöry ${ }^{1}$ \\ 1. AKH Linz, Institute of Radiology, Austria. 2. AKH Linz, Department of Neurology, Austria.
}

Correspondence: Franz A. Fellner, M.D. Address: AKH Linz, Institute of Radiology Krankenhausstr. 9, A-4020 Linz, Austria. E-Mail: franz.fellner@akh.linz.at

Received: November 28, 2013

Accepted: December 19, 2013

Online Published: December 24, 2013

DOI : $10.5430 / j b g c . v 4 n 2 p 1$

URL: http://dx.doi.org/10.5430/jbgc.v4n2p1

\begin{abstract}
Purpose: The mismatch concept in stroke magnetic resonance (MR) is based on the assumption that diffusion weighted imaging (DWI) indicates infarct core representing irreversibly damaged tissue. However, this thesis has not been proven yet in a large patient cohort. On the other hand, some publications report cases with possible remission of lesions at DWI. Thus, the aim of this study was to analyze temporal evolution of restricted diffusion at follow up MR imaging and analyze clinical and imaging features of cases with remission of DWI lesions.
\end{abstract}

Methods and Materials: 176 consecutive stroke patients (within 12 months) demonstrating restricted diffusion at initial MR examination were evaluated. Extension of diffusion restriction was judged both on initial MR as well as on follow-up after 24 hours. Changes in lesion extension were assessed by two experienced neuroradiologists in consensus.

Results: Extension of lesions with restricted diffusion at follow up MR was identical to the initial scan in 104/176 patients (59.1\%) and increased in 66/176 (37.5\%). The lesion size was decreased (meaning partial remission of the lesion) in 6/176 (3.4\%); none of the lesions showed complete remission. All six patients with partial remission of DWI lesions underwent early i.v. thrombolysis and demonstrated excellent clinical outcome. In one case out of the six cases with remission of DWI lesions, the DWI lesions demonstrated a re-increase in size after the first follow up examination, seen at late follow-up.

Conclusion: Our findings prove the existing assumption that diffusion restriction represents the infarct core, which may remain unchanged or increase in volume over time, indicating an accuracy of almost 97\%. Partial resolution of DWI lesions is extremely rare and usually associated with early i.v. thrombolysis and favourable clinical outcome.

\section{Key words}

Magnetic resonance, Stroke, Diffusion-weighted imaging, Infarction core

\section{I ntroduction}

In the state-of-the-art management of stroke patients, magnetic resonance imaging (MRI) including diffusion-weighted imaging (DWI) is considered to be the clearly superior imaging modality for the early diagnosis of ischemic brain infarction. Furthermore, stroke-MR serves as a basis for therapy decision and management. The role of diffusion weighted MR imaging has been underlined by numerous papers, recently by Lovblad et al. $2009^{[1]}$. 
The underlying assumption for clinical stroke management is that DWI lesions represent the final infarct core (meaning unsalvageable brain tissue), whereas the perfusion-weighted imaging lesion - exceeding the area of the DWI lesion defines the penumbra (potentially salvageable brain tissue) ${ }^{[2]}$. For clinical practice, this assumption has proven useful since in earlier studies, DWI lesions showed good correlation with final infarct core size seen on CT or T2W MR images ${ }^{[1]}$. However, several exceptions to this pattern were described over the last years, where reversal of DWI lesions has been found to a certain degree and under certain conditions. In this context, possible reversal of DWI lesions has been shown in cases of early vessel recanalization and the possibility of reversal of DWI lesions has been correlated with the degree of ADC signal drop. However, one paper ${ }^{[3]}$ supported the theory that even DWI lesions with severe ADC decrease do not definitely predict irreversible tissue damage. Taken together however, the mentioned literature about possible reversal of DWI lesions is limited and few data are available about the short term course of diffusion lesions, particularly within the first days after stroke onset with multiple MR follow up examinations. In addition there is no data about the course of diffusion lesion in a larger subsequent patient cohort. To our knowledge, only one paper investigated course of DWI lesions with short and longer term follow up to date ${ }^{[4]}$.

Thus, the aim of this paper was to assess the temporal evolution of DWI lesions at short term follow up to evaluate the accuracy of DWI to be indeed a marker of non salvageable brain tissue. In addition, correlation to final infarct core size in the chronic stage after brain infarction was performed. Furthermore, cases with remission of diffusion lesions were analysed in terms of their clinical and imaging features.

\section{Materials and methods}

\subsection{Study design and inclusion criteria}

A prospective study over a 12 months period was set up. Within this time interval, all patients with suspicion of acute stroke, referred to acute stroke MR imaging were analyzed. Patients who were shown to have ischemic lesions using DWI at the initial acute stroke MR examination and were established final diagnosis of ischemic brain infarction with further clinical and neurological workup were included.

Exclusion criteria were patients with unsure or absent DWI lesions and patients with imaging or clinical diagnosis of any other cerebral pathology. Patients with missing follow-up MRI on the first day after stroke onset or inadequate image quality were excluded also.

After initial MRI, all patients included into the study were referred for MRI follow up at the following day. This study was performed within a time interval from 16 hours to 40 hours after the initial examination. For a subset of patients, late follow up examinations were available (CT and/or MRI) - this was available according to the clinical need.

\subsection{Data acquisition}

All MR examinations were performed on a 1.5 T MR unit (Magnetom Avanto / Magnetom Symphony, Siemens Medical Solutions, Germany) with a standard head and neck coil.

The MR protocol for initial (day 0) acute stroke MR and day 1-follow ups included: DWI, FLAIR, T2 ${ }^{*}$-GRE, 3D-TOF, PWI and T1w-SE post contrast agent. Day 0 examinations additionally included contrast enhanced MR-angiography of the neck vessels.

DWI was performed as a single shot EPI sequence with whole brain coverage using a slice thickness of $6 \mathrm{~mm}$ and a pixel size $1.1 \mathrm{~mm} \times 1.1 \mathrm{~mm}$, TR $4300 \mathrm{~ms}$, TE $107 \mathrm{~ms}$, time of acquisition was one minute and six seconds. 
MR protocol for later follow up examinations included: DWI, FLAIR, T1w-SE, T2w-TSE, T2 ${ }^{*}$-GRE, post contrast material T1w-SE and a 3D T1w-GRE-dataset.

\subsection{Data analysis}

All imaging studies were read by two experienced neuroradiologists (FAF, DF) in consensus.

DWI lesions were assessed visually, thereby all lesions with notably increased signal on $b=1,000\left(\mathrm{~mm} / \mathrm{s}^{2}\right)$ signal and consecutive signal loss on the ADC maps were called positive.

Visual evaluation of DWI lesions was performed in day 0 and day 1 examinations regarding volume of lesions. Lesions were classified according to progression, no change, partial remission and complete remission at day 1 (4-tier scale). DWI lesions were correlated visually with chronic infarct size on late (later than day 1) follow- up examinations (MRI and CT), where available. In cases with remission of DWI lesions, clinical data and clinical course were analyzed.

\section{Results}

Within the study period, a total of 450 patients with acute stroke symptoms were examined with our MR stroke protocol on day 0 and day 1 . From this population, 176 patients remained meeting the inclusion criteria and were included into the study. The DWI lesions seen in these patients were lacunar infarction $(n=65)$, partial territorial ischaemia $(n=102)$ and territorial ischaemia $(n=9)$.

In all patients, day 1 follow ups of adequate quality were available (time range 16 hours to 40 hours after initial MRI). Treatment after diagnosis was i.v. thrombolysis in the majority of patients $(n=144)$, and conservative treatment in 32 patients.

Concerning the temporal course of DWI lesions, complete remission of DWI lesions was not seen in any patient. Six patients (3.4\%) demonstrated partial remission of the initial DWI lesion, their clinical and imaging features are summarized in the table. 104 lesions were stable at follow up (59.1\%), and 66 lesions (37.5\%) showed progression to a variable degree.

All six patients with partial remission of DWI lesions were patients with partial territorial infarction. In addition, the extent of the DWI lesions was correlated with the perfusion lesions in these patients. All DWI lesions were covered to an extent greater than $50 \%$ by the PWI lesions.

Figure 1. A fifty-nine years old male patient, presenting with acute left side hemiparesis

First row: Initial (day 0) MRI: a. DWI b-value, b. DWI ADC, c. FLAIR. DWI (a, b) demonstrates a lesion with restricted diffusion at the level of the corona radiata on the right side with FLAIR (c) being negative for a demarcated lesion.

Second row: MRI day 1: d. DWI b-value, e. DWI ADC, f. FLAIR. There is significant remission of the size of the DWI lesion. FLAIR demonstrates only a faintly demarcated lesion in correlation with day1 DWI lesion size.
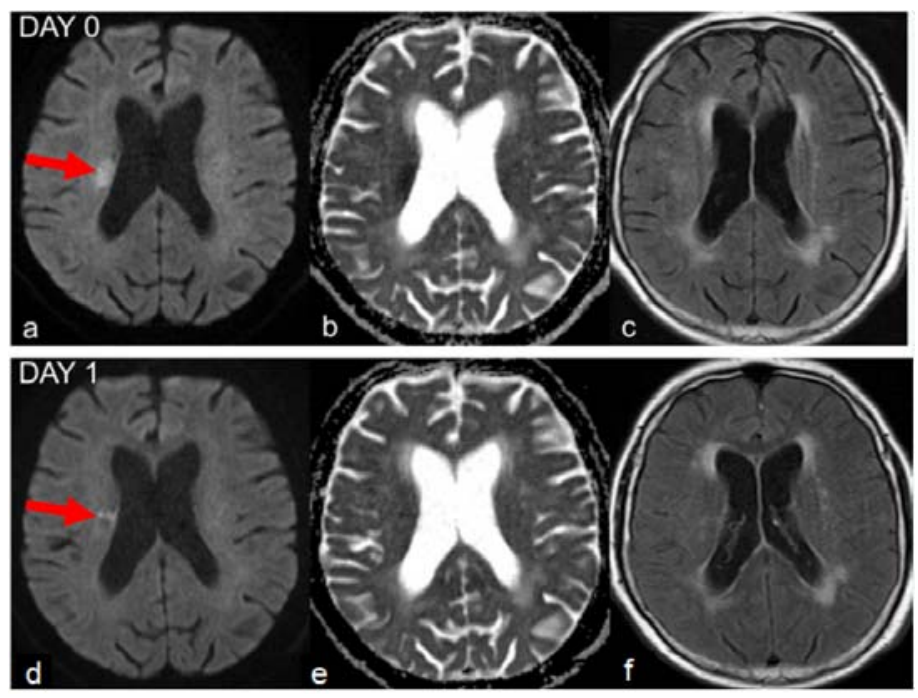

Published by Sciedu Press 
Furthermore, all six patients underwent early i.v. thrombolysis within four hours after onset of the symptoms and all patients demonstrated favourable clinical outcome (see Table). Three of six patients had late follow up examinations (MRI $\mathrm{n}=2$, CT $\mathrm{n}=1$; seven days, 37 days and three months after initial examination). In two out of three patients, the size of the day 1 DWI lesion was confirmed at late follow-up (see Figure 1 and Figure 2).

Table. Clinical characteristics of patients with (partial) remission of DWI lesions

\begin{tabular}{llllll}
\hline Patient & Onset to needle & NIH day $\mathbf{~}$ & $\begin{array}{l}\text { NIH at } \\
\text { discharge }\end{array}$ & $\begin{array}{l}\text { Vessel occlusion/ } \\
\text { recanalization }\end{array}$ & PWI lesion/mismatch \\
\hline 1 & $1: 35$ & 4 & 0 & Yes/yes & Yes/yes \\
2 & $2: 15$ & 9 & 0 & No & Yes/no \\
3 & $2: 25$ & 6 & 0 & No & Yes/yes \\
4 & $2: 50$ & 8 & 0 & No & Yes/no \\
5 & $3: 38$ & 5 & 0 & No & Yes/yes \\
6 & $2: 00$ & 10 & 0 & No & Yes/yes \\
\hline
\end{tabular}

Note. Onset to needle: Time interval between onset of symptoms and beginning of i.v. thrombolysis (hours:minutes); NIH day 0 resp. at charge: NIH score initial and at discharge;

Vessel occlusion/recanalization: Presence of vessel occlusion at TOF-Angiography at initial MRI and at day 1 follow up; PWI lesion/mismatch: Presence of a PWI lesion and of a mismatch $>20 \%$ at initial MRI.

Figure 2. A sixty-three years old male patient, presenting with left sided hemiparesis and facial palsy

First row: Initial (day 0) MRI: a. DWI b-value, b. DWI ADC, c. FLAIR. DWI (a, b) demonstrates a lesion with restricted diffusion located in the right MCA territory. Note. a pre-existing PMID (presumed microvascular disease) lesion (chronic infarction) (arrow head) is shown in FLAIR (c) adjacent to the diffusion lesion, however that is not connected in any way with the acute stroke.

Second row: MRI day 1: d. DWI b-value, e. DWI ADC, f. FLAIR. $27 \mathrm{~h}$ after initial MRI. The patient underwent i.v. thrombolysis 200 minutes after stroke onset.

Significant remission of the size of the lesions at DWI is shown, FLAIR is negative for demarcated ischemia.
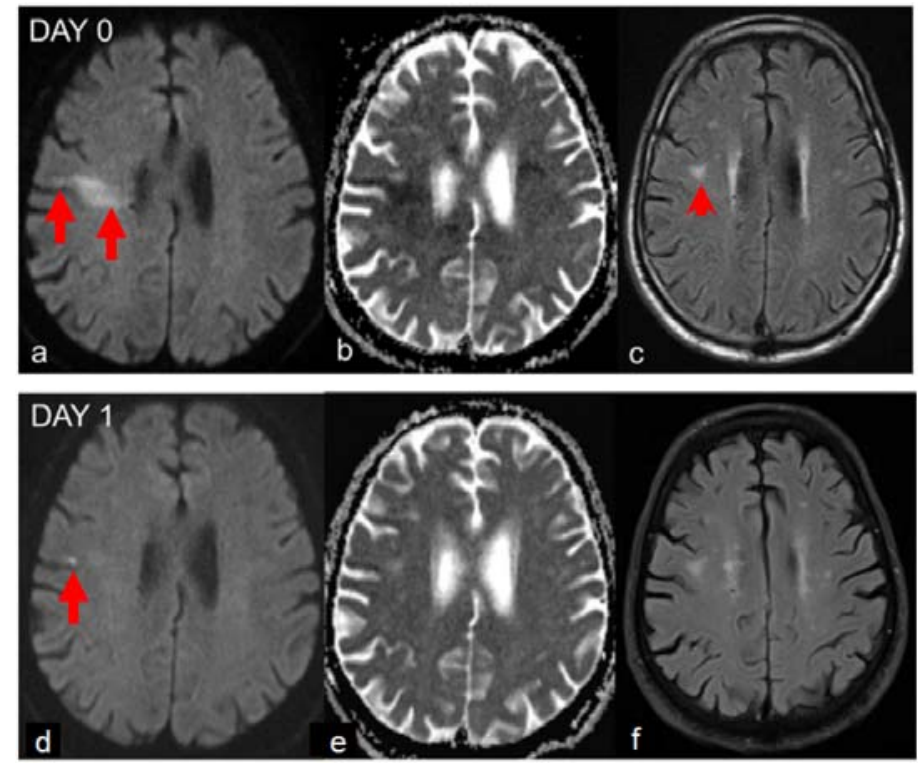

In one patient, the final infarct size was smaller than the day 0 lesions, but somewhat larger than the day 1 lesions meaning a slight re-growth of the lesion after the initial remission (see Figure 3).

\section{Discussion}

Unquestionably, stroke MR has evolved as the superior imaging modality for the workup of patients with suspected stroke, providing thereby the necessary information for treatment decision and patient management. This however, is based on the assumption that the volume of the DWI lesion at initial MRI truly represents unsalvageable brain tissue and that the PWI lesion demonstrates the potentially salvageable penumbra. Despite the fact that this assumption is supported largely by clinical evidence, there have been several publications within the last decade concerning possible reversal of DWI lesions under certain conditions ${ }^{[3-6]}$. However, the existing papers are very heterogeneous, particularly in clinical characteristics of patients and follow up settings. Taken together, the data on frequency and features of cases with remission of DWI 
lesions is unsatisfactory. Nevertheless, there is now an unsatisfying uncertainty among physicians in stroke therapy, whether DWI truly represents infarct core.

Figure 3. A seventy-three years old female patient presenting with right-sided hemiparesis of the lower limb.

First row: DWI (b-value) at the initial MRI (day 0) demonstrates a lesion with restricted diffusion in the MCA territory at the left side.

Second row: DWI (b-value) at day 1 follow-up MRI demonstrates a DWI lesion which is clearly smaller than the lesion seen at initial MRI.

Third row: FLAIR imaging 37 days after stroke onset demonstrates a post-ischemic lesion that is smaller than the DWI lesion at day0 but somewhat larger than day 1.

DWI lesion - meaning a slight re-growth of infarction core between day 1 and late follow up.

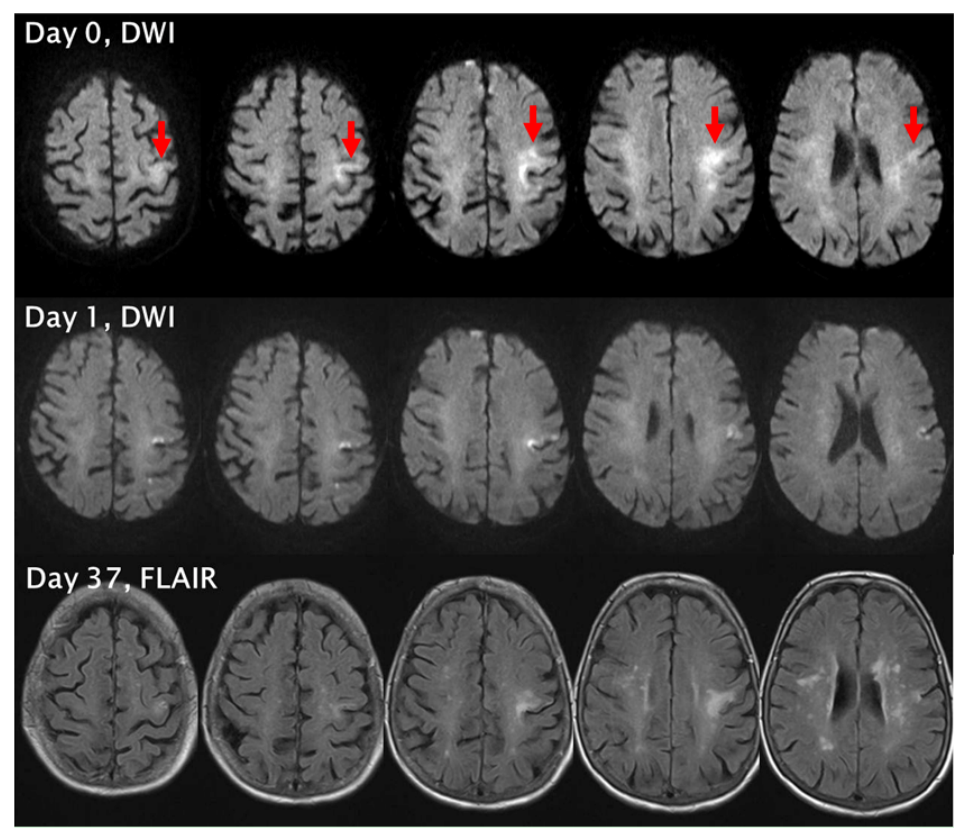

With regard to this situation, the aims of the present study were as follows:

- First, to evaluate if DWI is a reliable marker for non salvageable brain tissue (proven by follow-up studies).

- Second, to assess the course of diffusion lesions at short term follow up and correlate size of DWI lesions with final infarct size.

- Third, to analyse patients with reversal of DWI lesions in regard to clinical data.

In this patient series, partial reversal of DWI lesions was seen in $3.4 \%$ only; complete remission was not seen in any case. The remaining $96.6 \%$ of lesions demonstrated either stability or further growth at follow up. This data demonstrate that DWI can be regarded as a reliable marker for infarct core in the vast majority of cases and that reversal of DWI lesions is a very rare event.

Only a small number of papers in the literature evaluated the rate of patients with DWI remission in a cohort of stroke patients. To our knowledge, only one group performed a similar study setting to ours ${ }^{[4]}$ : In a series of 15 stroke patients, four demonstrated partial reversal of DWI lesions. Since this is equivalent to $26.7 \%$, reversal to DWI lesions was seen significantly more often by this group than in our series. Only one of the patients reported showed a minimal secondary increase of the size of the DWI Lesions on a day 7 follow up.

Olivot et al. ${ }^{[5,6]}$ investigated 32 acute stroke patients that received early i.v. thrombolysis in a study with follow up imaging three hours to 6 hours after thrombolysis and after 30 days. The authors reported an overall median volume decrease of DWI lesions of 43\%, however, no absolute numbers of patients with DWI reversal are given in this paper.

In summary, the literature is particularly heterogeneous in regard to the time point of the performed follow up examinations. Olivot et al. ${ }^{[5,6]}$ who performed a very early MRI follow up after treatment found a more frequent DWI reversal than papers with follow ups on the next day, as in our case. Since very little is known about the precise temporal evolution of DWI lesions in hours after stroke onset, the time point of follow up may here have a certain effect on the size of DWI lesion found at a given time point. 
Characteristics of our patient series was a very short median time interval between onset of the symptoms and the beginning of the MRI study and a very high proportion of patients receiving i.v. thrombolysis ( $\mathrm{n}=144 \mathrm{vs}$. conservative treatment in 32 cases.)

In one of six patients with partial remission of the DWI lesion, a secondary re-increase in size of the lesion was seen between day1 follow up and a late follow up on day 37. This phenomenon has already been described in the literature in a series by Kidwell ${ }^{[4]}$ et al. who described seven cases with remission of DWI lesions of which showed a secondary re-increase in size between a day 1 and day 7 MRI study. However, the observed patient cohort was different from ours; all patients had initial large artery occlusion and achievement of vessel recanalization during therapy ${ }^{[4]}$. In a series by Fiehler et al. ${ }^{[3]}$, secondary re-increase of the DWI lesion was observed in one patient.

There were very similar clinical characteristics of the six patients with partial remission of the DWI lesions: All six patients had partial territorial infarction and received early i.v. thrombolysis within four hours after onset of the symptoms; In addition, all patients achieved excellent clinical outcome with either complete or nearly complete remission of the clinical symptoms (see Table). This is in good correlation with other findings that supported the assumption that very early vessel recanalization is crucial for favourable patient outcome ${ }^{[7]}$.

Two papers ${ }^{[5,6]}$ investigated whether presence and patterns of coexisting PWI lesions affect the probability of DWI lesions reversal. They concluded that DWI lesions with absent coexisting PWI lesions and DWI lesion with an extent greater than 50\% not covered by a PWI lesion would have a higher probability for reversal. In our patients with DWI reversal, all patients had coexisting PWI lesions and all DWI lesions were covered to an extent greater than $50 \%$ by the PWI lesion - so we could not support these findings in our data.

Other groups demonstrated a correlation between severity of ADC decrease and probability of DWI reversal suggesting that DWI lesions with higher ADC values are more likely to reverse ${ }^{[5,6]}$. However, one paper ${ }^{[4]}$ found that even lesions with severe ADC decrease do not necessarily represent unsalvageable brain tissue. Quantitative evaluation of ADC values was not performed in our study to date.

Our study had following limitations: Firstly, correlative ADC measurements were not taken into account given the evidence that severity of lesions ADC value decrease may influence probability of DWI lesion reversal. Secondly, evaluation of DWI maps was performed only visually on 2D maps with no quantitative threshold and no volumetric data. However, our study design represents clinical routine and we believe visual assessment does not compromise the results presented here. Thirdly, in three patients a long term follow up was not availabe, so correlation with final infarct sizes could not be assessed in these patients. The precise clinical correlation of imaging findings and long term clinical patient outcome was not aim of this work and will be addressed in a distinct paper.

In conclusion, our data demonstrate that reversal of DWI lesions is a very rare event and that DWI can be regarded a clinically reliable marker for non-salvageable brain tissue.

\section{References}

[1] Lövblad KO, Altrichter S, Viallon M, Sztajzel R, Delavelle J, Vargas MI, et al. Neuro-imaging of cerebral ischemic stroke. J Neuroradiol. 2008; 35(4): 197-209. PMid: 18329713. http://dx.doi.org/10.1016/j.neurad.2008.01.002

[2] Schellinger PD, Bryan RN, Caplan LR. Evidence-based guideline: The role of diffusion and perfusion MRI for the diagnosis of acute ischemic stroke: report of the Therapeutics and Technology Assessment Subcommittee of the American Academy of Neurology. Neurology. 2010 Jul 13; 75(2): 177-85. PMid: 20625171. http://dx.doi.org/10.1212/WNL.0b013e3181e7c9dd

[3] Fiehler J, Foth M, Kucinski T. Severe ADC decreases do not predict irreversible tissue damage in humans. Stroke. 2002 Jan; 33(1): 79-86. PMid: 11779893. http://dx.doi.org/10.1161/hs0102.100884 
[4] Kidwell CS, Saver JL, Mattiello J. Thrombolytic reversal of acute human cerebral ischemic injury shown by diffusion/perfusion magnetic resonance imaging. Ann Neurol. 2000 Apr; 47(4): 462-9. http://dx.doi.org/10.1002/1531-8249(200004)47:4<462::AID-ANA9>3.0.CO;2-Y

[5] Olivot JM, Mlynash M, Thijs VN. Geography, structure, and evolution of diffusion and perfusion lesions in Diffusion and perfusion imaging. Evaluation for Understanding Stroke Evolution (DEFUSE). Stroke. 2009 Oct; 40(10): 3245-51. PMid: 19679845. http://dx.doi.org/10.1161/STROKEAHA.109.558635

[6] Olivot JM, Mlynash M, Thijs VN. Relationships between cerebral perfusion and reversibility of acute diffusion lesions in DEFUSE: insights from RADAR. Stroke. 2009 May; 40(5): 1692-7. PMid: 19299632. http://dx.doi.org/10.1161/STROKEAHA.108.538082

[7] Neumann-Haefelin T, Du Mesnil de Rochemont R, Fiebach JB. Effect of incomplete (spontaneous and postthrombolytic) recanalization after middle cerebral artery occlusion: a magnetic resonance imaging study. Stroke. 2004 Jan; 35(1): 109-14. PMid: 14671246. http://dx.doi.org/10.1161/01.STR.0000106482.31425.D1 